\title{
A NORMAL FORM THEOREM FOR LATTICES COMPLETELY GENERATED BY A SUBSET
}

\author{
GEORGE GRÄTZER AND DAVID KELLY ${ }^{1}$
}

\begin{abstract}
For an $\mathfrak{m}$-complete lattice $L$ ( $\mathfrak{m}$ is an infinite regular cardinal) and subset $X$ of $L$ that m-generates $L$, we prove a Normal Form Theorem for elements of $L$ expressed as polynomials over $X$. This generalizes a theorem of B. Jonsson in which such a representation is found for the lattice $L$ freely m-generated by a poset $X$. We also apply this result to free m-products of $m$-complete lattices.
\end{abstract}

Throughout this paper, $m$ is an infinite regular cardinal. A lattice $L$ is m-complete (or $L$ is an $\mathfrak{m}$-lattice) iff for any nonempty $S \subseteq L$ with $|S|<\mathfrak{m}$, the join and meet of $S$ exist in $L{ }^{2}$ An m-lattice $L$ is m-generated by a set $X$ iff $L=X_{\mathfrak{m}}$, where the subsets $X_{\alpha}$ of $L(\alpha \leqslant \mathfrak{m})$ are inductively defined by: $X_{0}=X$; for $\alpha>0, X_{\alpha}$ consists of all elements of the form $\bigvee S$ or $\wedge S$ where $S \subseteq \cup\left(X_{\beta} \mid \beta<\alpha\right)$ and $0<|S|<\mathfrak{m}$. (Equivalently, $L$ is the smallest $\mathrm{m}-$ sublattice of $L$ that contains $X$.)

Henceforth, $L$ and $X$ are as above. The rank of $a \in L$ (with respect to $X$ ), denoted by $\rho(a)$, is the least $\alpha$ such that $a \in X_{\alpha}$. Whenever we write $\bigvee T$, it is to be understood that $T \subseteq L$ and $0<|T|<\mathfrak{m}$. An element $a \in L$ is strictly m-join-reducible (with respect to $X$ ) iff $a=\bigvee T$ for some $T \subseteq L$ and $\rho(t)<\rho(a)$ for all $t \in T$; in this case, the representation $a=\bigvee T$ is called strict. Any element that is not strictly m-join-reducible is called strictly $\mathrm{m}$-join-irreducible. We shall also use the dual concepts.

Every element of $X$ is strictly m-join-irreducible and strictly m-meetirreducible. Obviously, every element of $L-X$ is strictly m-join-reducible or strictly $\mathrm{m}$-meet-reducible. Our main result is the

Normal Form TheOREM. Let $L$ be an m-lattice that is m-generated by the set $X$. If $a \in L$ is strictly m-join-reducible, then there is a strict representation $a=\bigvee T$ such that

(i) every element of $T$ is strictly m-join-irreducible;

(ii) if $t=\wedge S$ is a strict representation of some $t \in T$, then $s \nless a$ for all $s \in S$.

Received by the editors November 17, 1976.

AMS (MOS) subject classifications (1970). Primary 06A23.

Key words and phrases. Complete lattice, representation, join-irreducible.

'The research of both authors was supported by the National Research Council of Canada.

2Our usage of the term " $m$-complete" is different from the customary usage in the literature. 
1. Applications. Before coming to the proof of the Normal Form Theorem, we will give two of its corollaries. An element $a$ of an m-lattice $L$ is m-join-reducible iff it has a representation $a=\bigvee T$ with $a \notin T$. The first corollary, due to B. Jónsson [3], motivated the present paper.

COROLlaRY 1. Let $L$ be the completely free $m$-lattice generated by the poset $X$. If $a \in L$ is m-join-reducible, then there is a representation $a=\bigvee T$ such that

(i) every element of $T$ is m-join-irreducible;

(ii) if $t \in T-X$, then there is a representation $t=\wedge S$ such that $s \nless a$ for all $s \in S$.

Proof. By P. Crawley and R. A. Dean [1], no element of $L$ can be both $\mathfrak{m}$-join-reducible and $\mathfrak{m}$-meet-reducible, and every element of $X$ is $\mathfrak{m}$-joinirreducible. Therefore, $m$-join-reducible and strictly $m$-join-reducible are equivalent properties in $L$.

Corollary 2. Let $L$ be a free m-product of the family $\left(L_{i} \mid i \in I\right)$ of m-complete lattices, and set $X=\cup\left(L_{i} \mid i \in I\right)$, a subset of $L$. If $a \in L-X$ is $\mathfrak{m}$-join-reducible, then there is a representation $a=\bigvee T$ such that

(i) every element of $T-X$ is m-join-irreducible;

(ii) if $t \in T-X$, then there is a representation $t=\wedge S$ such that $s \nless a$ for all $s \in S$;

(iii) if $f^{3} t \leqslant a_{(i)}$ for $t \in T$ and $i \in I$, then $t=a_{(i)}$.

Proof. By G. Grätzer and D. Kelly [2], no element of $L-X$ can be both $m$-join-reducible and $m$-meet-reducible. Therefore, $m$-join-reducible and strictly $m$-join-reducible are equivalent properties in $L-X$. Let $a=\bigvee T_{0}$ be a strict representation satisfying the conditions of the theorem. For each $t \in T_{0}$ that satisfies $t \leqslant a_{(i)}$ for some $i \in I$, replace $t$ in $T_{0}$ by $a_{(i)}$, to form $T$. Then $a=\bigvee T$ and this is a representation satisfying the conditions of the corollary.

In [2], the second corollary is applied to show that the free m-product construction preserves semidistributivity. Similarly as in [3], we can prove a minimal property of the representation in Corollary 2. (We use the Structure Theorem of [2] in the proof.)

Proposition. Let $L, X$ and $a=\bigvee T$ be as in Corollary 2. If $a=\bigvee T^{\prime}$ is any representation, then for each $t \in T-X$ there exists $t^{\prime} \in T^{\prime}$ with $t \leqslant t^{\prime}$.

Proof. Let $t \in T-X$ and $t=\bigwedge S$ be a representation that satisfies (ii) of Corollary 2, and consider the valid inequality $\wedge S \leqslant \bigvee T^{\prime}$. By (iii), ${ }^{4}(C)$ cannot apply. Also, by (ii), $(\wedge W)$ cannot apply, leaving only $\left(W_{\vee}\right)$; this means that $t \leqslant t^{\prime}$ for some $t^{\prime} \in T^{\prime}$.

The reader may find it of interest to compare Corollary 2 with the minimal

${ }^{3} a_{(i)}$ is the lower cover of $a$ in $L_{i}$, see [2].

${ }^{4}(C),(\wedge W),\left(W_{\bigvee}\right)$ refer to conditions in the Structure Theorem of [2]. 
form theorem for free products in the finitary case, see H. Lakser [4].

2. Proof of the theorem. It is easily shown by induction on the rank that for any strictly $m$-join-reducible element $a$ of $L$, there is $T \subseteq L$ such that $a=\bigvee T$ is a strict representation and (i) holds. From now on, $a$ is a fixed strictly m-join-reducible element of $L$.

Following B. Jónsson [3], for $R \subseteq L, C(R)$ denotes the set of elements $b \in L$ such that $b \in R$ or $b \leqslant \bigvee T$, where $T \subseteq R$ and $\rho(t)<\rho(b)$ for all $t \in T$. For $R, R^{\prime} \subseteq L, R<R^{\prime}$ will mean that $R \subseteq C\left(R^{\prime}\right)$. Since $C(C(R))$ $=C(R)$, the relation $<$ quasi-orders the class of all subsets of $L$.

LEMMA. Let $C$ be a chain (with respect to inclusion) of subsets of $L$ whose

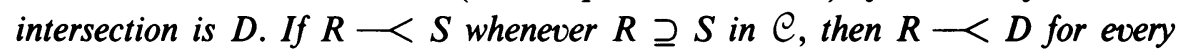
$R \in \mathcal{C}$.

Proof. Let $b \in R$ and $R \in \mathcal{C}$. We show that $b \in C(D)$ by induction on $\rho(b)$. We can assume that $b \notin D$. Thus, $b \notin S$ for some $S \in \mathcal{C}$. Therefore, $R \supset S$; this implies that $R<S$. Hence, $b \in C(S)$ and $b \leqslant \bigvee T$ for some $T \subseteq S$ such that $\rho(t)<\rho(b)$ for all $t \in T$. By induction, $T \subseteq C(D)$, and therefore, $b \in C(C(D))=C(D)$.

We now return to the proof of the theorem. Let $A$ be the set of elements $b$ of $L$ such that $\rho(b)<\rho(a), b \leqslant a$, and $b$ is strictly m-join-irreducible. Let $\mathscr{K}$ be the class of all subsets $R$ of $L$ such that $R \longrightarrow R^{\prime}$ implies that $R=R^{\prime}$ whenever $R^{\prime} \subseteq R$.

We define a sequence of subsets of $A$. Let $R_{0}=A$. If $R_{\alpha}$ is defined and $R_{\alpha} \in \mathscr{K}$, we terminate the sequence and set $R=R_{\alpha}$; if $R_{\alpha} \notin \mathscr{K}$, then choose $b \in R_{\alpha}$ such that $R_{\alpha}<\left(R_{\alpha}-\{b\}\right)$ and set $R_{\alpha+1}=R_{\alpha}-\{b\}$. If the $R_{\beta}$ have been defined for $\beta<\alpha$, where $\alpha$ is a limit ordinal, then we define $R_{\alpha}=\cap\left(R_{\beta} \mid \beta<\alpha\right)$. By the Lemma, $A<R_{\alpha}$ whenever $R_{\alpha}$ is defined. Since the sequence is strictly decreasing, it must terminate; thus $R \in \mathscr{K}$ is defined and $A<R$.

It is clear that $R$ satisfies condition (i) of the theorem. Assuming that (ii) fails, there is $r \in R$ and a strict representation $r=\bigwedge S$ such that $s \leqslant a$ for some $s \in S$. If $s$ is strictly m-join-reducible, let $s=\bigvee B$ be a strict representation such that (i) holds; otherwise, $B=\{s\}$. In either case, $B \subseteq A$. Thus, $B<R$ and since $\rho(b)<\rho(r)$ for all $b \in B$, we conclude that $B<(R-$ $\{r\})$. It follows that $R<(R-\{r\})$, which contradicts $R \in \mathcal{K}$, concluding the proof that $R$ satisfies (ii).

We have already observed that there is $A_{0} \subseteq A$ with $0<\left|A_{0}\right|<\mathrm{m}$ such that $a=\bigvee A_{0}$. Thus there is $T \subseteq R$ with $0<|T|<\mathrm{m}$ such that $A_{0}<T$. Clearly, $a=\bigvee T$ and this representation satisfies all the conditions of the theorem.

\section{REFERENCES}

1. P. Crawley and R. A. Dean, Free lattices with infinite operations, Trans. Amer. Math. Soc. 92 (1959), 35-47. 
2. G. Grätzer and D. Kelly, Free m-products of lattices (to appear). See also Notices Amer. Math. Soc. 24 (1977), A-1, A-221, A-287.

3. B. Jónsson, Arithmetic properties of freely a-generated lattices, Canad. J. Math. 14 (1962), 476-481.

4. H. Lakser, Normal and canonical representations in free products of lattices, Canad. J. Math. 2 (1970), 394-402.

Departmient of Mathematics, University of Manttoba, Winnipeg, Manitoba, Canada R3T 2N2 